\title{
PATTERN OF ANTIMICROBIAL USE IN PEDIATRIC PATIENTS HOSPITALIZED IN TERTIARY LEVEL CARE HOSPITALS IN GREECE
}

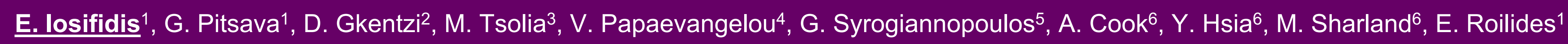

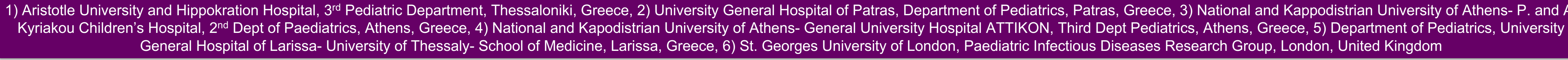

\section{BACKROUND}

Pediatric antimicrobial stewardship programs require the knowledge of trends in antimicrobial prescriptions in hospitalized pediatric patients. ${ }^{1,2}$

The aim of this study was to assess the pattern of antimicrobia prescriptions in hospitalized pediatric patients in Greece.

\section{METHODS}

One-day point-prevalence survey was conducted in three consecutive semesters during the period of 2016-2017, as part o the Global Antibiotic Resistance, Prescribing and Efficacy in Neonates and Children (GARPEC) project. 2,3

Five tertiary level hospitals in four Greek cities participated. Patients that were on antimicrobial treatment at 08:00 am of the day of the survey were included.

\section{RESULTS}

In total, 176 antimicrobial courses (297 prescriptions ranging from 11 to 176 among hospitals) were analyzed.

Of all antimicrobial prescriptions, $80.1 \%$ were administered intravenously and $17.5 \%$ orally.

Most of the prescriptions were administered for communityacquired infections (CAls), followed by hospital-acquired infections (HAls) and medical/surgical prophylaxis (Figure 1)

CAls mAls Medical/Surgical prophylaxis Other

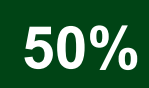

$29 \%$

$18 \%$

$4 \%$

Figure 1: Reason for antimicrobial administration

The most frequent antimicrobials were: $3^{\text {rd }}$ generation cephalosporins (18.5\%), penicillins (13.8\%) and aminoglycosides (13.1\%).
Antimicrobial courses were administered as monotherapy $(53.4 \%)$, co-administration of two (31.2\%), three $(9.6 \%)$ or at least four $(5.8 \%)$ antimicrobial agents (Figure 2).

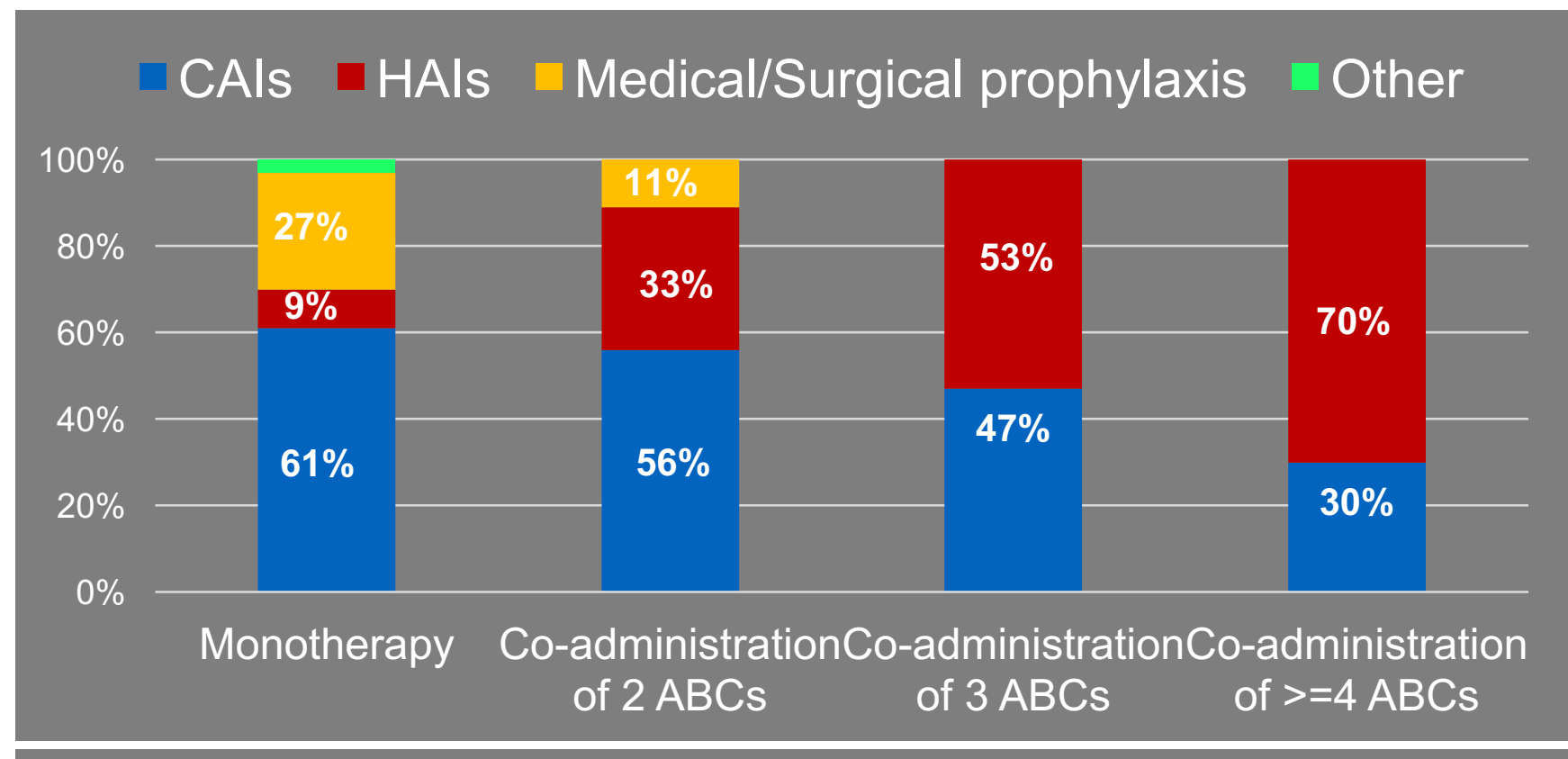

Figure 2: Antimicrobial courses

Monotherapy was used in 94 antimicrobial courses:

- The most common reason for prescribing one antimicrobia agent was CAls in $60.6 \%$, medical/ surgical prophylaxis in $26.5 \%$ and HAls in $9 \%$.

- Among CAls, urinary tract infection was the most common diagnosis. Ceftriaxone, cefotaxime and cefuroxime were frequently prescribed for both empirical and targeted treatment. In contrast, ampicillin, amikacin, co-trimoxazole and cefepime were used only when antimicrobial susceptibility tests were available.

- Community acquired bacterial lower respiratory tract infections (LRTIs) were the second most frequent reason for administration of monotherapy. For these cases the following antimicrobials were used: benzylpenicillin, ampicillin, ceftriaxone and clarithromycin

- Community acquired skin/soft tissue infections were the third most common reason for using monotherapy. Clindamycin was the most preferred antimicrobial agents in $50 \%$ of cases, followed by amoxicillin/clavulonate, cefuroxime and daptomycin.

- For medical prophylaxis, co-trimoxazole was the most commonly prescribed antimicrobial agent. The main reason for medical prophylaxis was chronic lung or renal disease.
Co-administration of two antimicrobials was used in 55 antimicrobial courses.

- The most frequent diagnosis was bacterial LRTIs. In community acquired LRTIs, ceftriaxone was combined with vancomycin, linezolid or clindamycin in most of the cases. In hospital acquired LRTIs different antimicrobials were used including ceftazidime/ amikacin, meropenem/vancomycin, piperacillin-tazobactam/ colistin, ciprofloxacin/cotrimoxazole, cefuroxime/ teicoplanin.

- Sepsis was also a frequent reason for co-administration of 2 antimicrobials. In community acquired sepsis, cefotaxime was combined with ampicillin. In hospital acquired sepsis, cefepime/vancomycin, meropenem/colistin were used.

- In community-acquired skin/soft tissue infections, clindamycin was combined with ceftriaxone, cefotaxime or amoxicillin/clavulonate.

- In community-acquired UTIs, amikacin was usually combined with ampicillin, ceftriaxone or cefotaxime.

Co-administration of 3 antimicrobials was found in 17 courses.

The main reason for antimicrobial use was bacterial LRTI or fever (most patients were neutropenic or mechanically ventilated).

- Aminoglycosides, cephalosporins and glycopeptides were frequently prescribed (13 different antimicrobial regimens).

Co-administration of at least 4 antimicrobials was found in

10 courses:

- In 8 courses, 4 antimicrobials were prescribed mainly for HAls including bloodstream infections/sepsis, mostly in neutropenic patients.

In two courses, 5 antimicrobials were administered in invasively ventilated children with chronic lung disease

\section{CONCLUSIONS}

- High rates of multiple antimicrobials, diverse combinations and intravenous administration was found in this survey.

- These findings constitute an appropriate target for antimicrobial stewardship programs. 